\title{
Design of Mechatronic Systems With Configuration-Dependent Dynamics: Simulation and Optimization
}

\author{
Maíra M. da Silva, Wim Desmet, and Hendrik Van Brussel, Fellow, IEEE
}

\begin{abstract}
This paper considers the simulation and optimization of mechatronic systems with configuration-dependent dynamics. A modeling methodology, able to capture the varying dynamics and the embedded control system actions, using affine reduced models and cosimulation, is proposed. In this way, mechatronic systems with configuration-dependent dynamics can be evaluated during the design phase. This methodology is applied to a pickand-place assembly robot and an experimental validation is carried out. The mechatronic design approach, which takes into consideration structural and control parameters, is considered. Using timedomain metrics, two control strategies are derived: a linear timeinvariant proportional-integral-derivative (PID) controller and a linear parameter-varying PID controller. Finally, design tradeoffs are evaluated in a truly mechatronic approach.
\end{abstract}

Index Terms-Computer-aided engineering, machine tools, optimization methods, simulation, time-varying systems.

\section{INTRODUCTION}

A LARGE number of mechatronic systems may have their eigenfrequencies and mode shapes dependent on the instantaneous spatial configuration, which inevitably affects the performance and the stability of the control system [1], [2]. In particular, mechatronic systems, such as machine tools, cartesian mechanisms, and pick-and-place machines, can be classified as systems with configuration-dependent dynamics since the relative motion between their flexible components can lead to time-varying boundary conditions. A typical example is a pick-and-place robot with a gripper carried by a flexible beam (see Fig. 1). Fast movements may excite the eigenfrequencies of the flexible beam, which are dependent on the beam length, causing vibrations and decreasing the positioning accuracy.

The demands for faster and more accurate machine tools, cartesian mechanisms, and pick-and-place machines, which can be classified as mechatronic systems with configurationdependent dynamics, are steadily increasing. These requirements are conflicting since fast movements may excite the machine eigenfrequencies, deteriorating its accuracy. Active control schemes are generally implemented to enhance the machine dynamic performance. Therefore, as the performance of active machines depends on both structural and control param-

Manuscript received January 1, 2008; revised June 18, 2008. Current version published December 17, 2008. Recommended by Technical Editor J. Desai. The work of M. M. da Silva was supported by the Brazilian Foundation Coordination for the Improvement of Higher Education Personnel, CAPES.

The authors are with the Department of Mechanical Engineering, Katholieke Universiteit Leuven, Celestijnenlaan 300 B, B-3001 Leuven, Belgium (e-mail: maira.dasilva@student.kuleuven.be).

Digital Object Identifier 10.1109/TMECH.2008.2003957

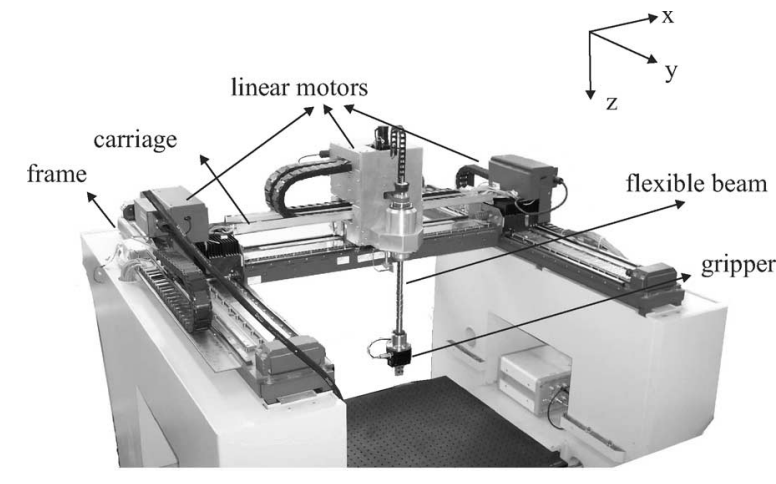

Fig. 1. Pick-and-place machine used as test case.

eters, their design should be performed along the lines of a mechatronic design approach [3]. The benefits of this approach, which deals with the integrated design of a mechanical system and its embedded control system, have been highlighted in several fields: motor-driven mechanisms design [4]-[6], machine tools design [7], active damping guide roller design [8], smart structures [9], active vibro-acoustic systems [10], and bioengineering [11], among others.

Simulation-based design has been extensively employed, not only for shortening development cycles and reducing costs, but also for enhancing product performance. According to the mechatronic design approach, simulation/optimization should be employed by the engineer as an effective design tool, enabling the direct access to structural and control parameters. Therefore, regarding the design of mechatronic systems with configurationdependent dynamics, two steps should be accomplished: 1) the simultaneous simulation of the mechanical system and the controller and 2) their integrated design/optimization.

The modeling of mechatronic systems with configurationdependent dynamics has been treated in several references [7], [12], [13]. The main difficulty during the modeling phase arises from the time-varying boundary conditions. The standard approach is the use of substructuring dynamic techniques, which are based on the assembly of component mode sets extracted from finite-element models using component-mode synthesis (CMS) for each configuration [7], [12]. Therefore, the machine can only be evaluated in discrete configurations. An integrated finite-element and multibody simulation has been proposed by [13]. This technique is relatively time consuming and no control integration is foreseen, which are important drawbacks.

There are mainly two control strategies that can be employed for systems with configuration-dependent dynamics: 1) robust 
linear time-invariant (LTI) controllers that can be explicitly designed to take into account the dynamical variations as uncertainties or (2) linear parameter-varying (LPV) controllers that can adapt according to the parameter variations. Modal LTI controllers have been applied to control configuration-dependent dynamics of a flexible-link manipulator [14] and a planar parallel platform [15]. A robust LTI controller and a gain-scheduling controller (LPV) have been designed for a pick-and-place assembly robot by [1] and [16]. Adaptive controllers for a two-link robot with a time-varying payload have been derived by [2].

This paper presents a methodology to model and simulate a mechatronic system with configuration-dependent dynamics, using cosimulation between a commercial multibody environment, LMS Virtual.Lab Motion, and Matlab/Simulink. Using the hereafter proposed approach, different control design techniques and optimization methodologies can be applied regarding the mechatronic system, and considering not only discrete configurations, but also continuous operation. In this way, structural and control parameters can be taken into consideration during the design phase.

In order to demonstrate the time-domain capabilities of the proposed modeling methodology, LTI and LPV control strategies are derived using time-domain metrics. However, the modeling methodology is not restricted to this control design approach, and can be employed to evaluate any control design technique, e.g., robust and gain-scheduling controllers.

The paper is organized as follows. Section II presents the proposed modeling methodology, able to capture not only the configuration-dependent dynamics, but also the control actions. A pick-and-place assembly robot was chosen as test case (see Fig. 1). Its setup and model are described and experimentally validated in Section III. Using time-domain metrics, LTI and LPV proportional-integral-derivative (PID) controllers are derived. Design tradeoffs are evaluated in a truly mechatronic approach, exemplifying the benefits of the integrated mechatronic design in Section IV. Finally, some conclusions are drawn.

\section{Modeling of Mechatronic Systems With CONFIGURATION-DEPENDENT DYNAMICS}

One way of modeling mechatronic systems is to use flexible multibody models and cosimulation schemes. In general, the controller is implemented in Matlab/Simulink, while the flexible multibody model is implemented in a multibody environment. However, a mechatronic systems with configuration-dependent dynamics requires a model able to capture this dependency. This can be performed, among others, by creating an updated finite-element model for each integration step or implementing a sliding joint for the flexible body connection [17]. Neither of these options are available in the standard commercial multibody environments.

To overcome this issue, a novel modeling methodology is proposed. First, the system is divided in two parts: 1) a subsystem whose dynamics do not depend on the configuration and (2) a subsystem with configuration-dependent dynamics. The former can be modeled using any commercial multibody package. The

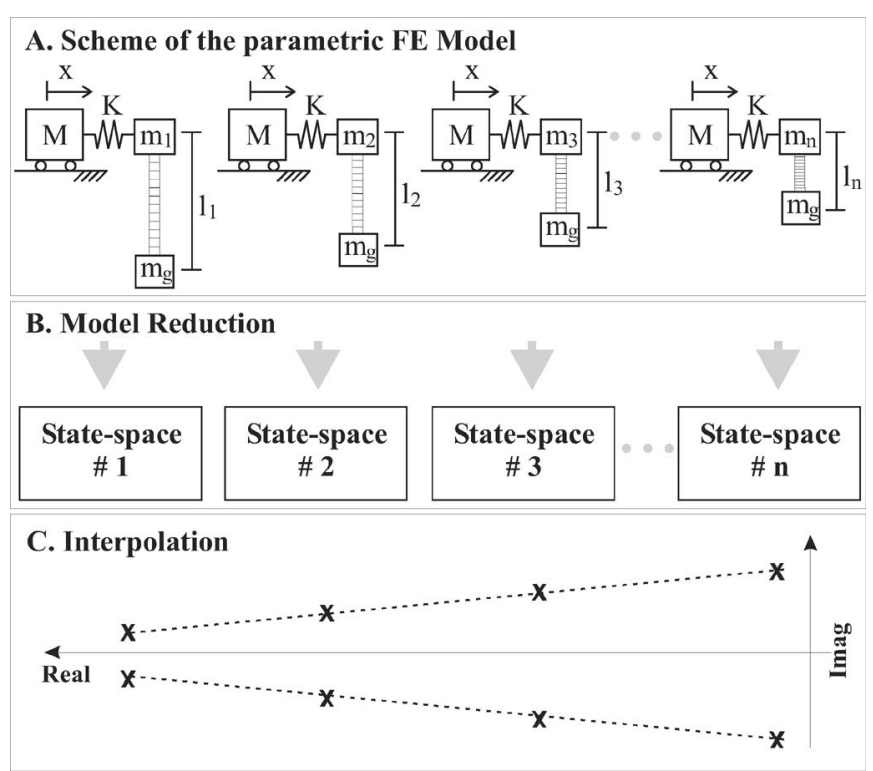

Fig. 2. Modeling of systems with configuration-dependent dynamics: threestep methodology.

latter can be modeled using an LPV model. Both can be coupled using cosimulation.

A three-step methodology (see Fig. 2) to model the subsystem with configuration-dependent dynamics, yielding an LPV model, is proposed.

1) A parametric high-order finite-element model is elaborated.

2) Local linear models are extracted at several discrete configurations using a linear model reduction technique.

3) An LPV state-space model is built by affine interpolation between poles, zeros, and gains extracted from reduced models in several discrete configurations. This LPV model can be implemented in Matlab/Simulink, which, for each integration step, reevaluates the state-space model depending on the parameter.

Each one of these steps is described in detail hereafter.

\section{A. Parametric Finite-Element Model}

A high-order finite-element model of the subsystem with configuration-dependent dynamics is, initially, created. Any commercial finite-element software can be used for deriving this model. Since several discrete models for different configurations are necessary, a good approach to generate these models is to create a parametric high-order finite-element model. This model can be described by a parameter varying second-order differential equation

$$
\mathbf{M}(\mathbf{l}) \ddot{\mathbf{q}}(\mathbf{l})+\mathbf{C}(\mathbf{l}) \dot{\mathbf{q}}(\mathbf{l})+\mathbf{K}(\mathbf{l}) \mathbf{q}(\mathbf{l})=\mathbf{L}(\mathbf{l}) \mathbf{f}(\mathbf{l})
$$

where $l$ is a vector of varying parameters, i.e., the configuration vector, $\mathbf{M}$ is the mass matrix, $\mathbf{C}$ is the damping matrix, $\mathbf{K}$ is the stiffness matrix, $\mathbf{q}$ are the physical degrees of freedom, $\mathbf{f}$ is the applied forces vector, and $\mathbf{L}$ is the input force influence matrix, indicating the way how the input forces act on the structure. 
The accuracy of the reduced models extracted from the parametric finite-element model relies on the accuracy of the highorder finite-element model, which may depend on the available information during the design phase and the chosen modelreduction technique. For a discrete configuration $i$, a model can be described evaluating (1) for $\mathbf{l}=\mathbf{l}_{i}$. Several discrete models can be extracted varying the parameter vector within its feasible range.

\section{B. Model Reduction}

The CMS provides an appropriate solution for the reduction of a finite-element model [18]. It is a form of substructure coupling analysis in which the dynamic behavior of each substructure is formulated as a superposition of modal contributions. This modal contribution consists of preselected component modes of the following types: normal modes, rigid body modes, constrained modes, attachment modes, inertia relief modes, and inertia relief attachment modes.

There are combinations of the rigid body modes, constrained modes, attachment modes, inertia relief modes, and inertia relief attachment modes that generate a superposition of the modes, sufficient to determine exactly the static response of a component submitted to external forces applied at boundary nodes. Any of them may be supplemented by dynamic modes: fixed interface, free interface, or hybrid interface defined by the normal modes [7].

For a discrete configuration $i$, the model can be described by a set of second order differential equations:

$$
\mathbf{M}\left(\mathbf{l}_{i}\right) \ddot{\mathbf{q}}\left(\mathbf{l}_{i}\right)+\mathbf{C}\left(\mathbf{l}_{i}\right) \dot{\mathbf{q}}\left(\mathbf{l}_{i}\right)+\mathbf{K}\left(\mathbf{l}_{i}\right) \mathbf{q}\left(\mathbf{l}_{i}\right)=\mathbf{L}\left(\mathbf{l}_{i}\right) \mathbf{f}\left(\mathbf{l}_{i}\right) .
$$

For the sake of brevity, any parameter-dependent function $h\left(\mathbf{l}_{i}\right)$ is represented just as $h$, in this section. In any CMS technique, the displacements of the physical coordinate $q$ are represented in terms of component coordinates $\boldsymbol{\eta}$ using the classical modal transformation:

$$
\mathbf{q}=\boldsymbol{\Psi} \eta
$$

where the transformation $\Psi$ consists of preselected component modes.

The reduced model is built using the Craig-Bampton method [18]. According to this method, the modal transformation $\Psi$, defined by (4), is composed by the constrained modes, $\Psi_{c}$, and the normal modes with fixed interface, $\boldsymbol{\Phi}_{k}$

$$
\boldsymbol{\Psi}=\left[\begin{array}{ll}
\boldsymbol{\Psi}_{c} & \boldsymbol{\Phi}_{k}
\end{array}\right] .
$$

Performing the modal transformation, the equation of motion can be written in component coordinates $\boldsymbol{\eta}$ [19].

$$
\ddot{\boldsymbol{\eta}}+2 \boldsymbol{\xi} \boldsymbol{\Omega} \dot{\boldsymbol{\eta}}+\boldsymbol{\Omega}^{2} \boldsymbol{\eta}=\boldsymbol{\mu}^{-1} \mathbf{\Psi}_{u}^{T} \mathbf{f}
$$

where $\Omega$ are the modal frequencies, $\boldsymbol{\xi}$ is the modal damping ratio, $\boldsymbol{\mu}$ is the modal mass, and $\boldsymbol{\Psi}_{u}=\mathbf{L}^{T} \boldsymbol{\Psi}$, where $\mathbf{L}$ is the force influence matrix [19]. The component coordinates are then represented by the degree of freedom $\boldsymbol{\theta}$, representing the actuators and the modal coordinates $\phi$

$$
\boldsymbol{\eta}=\left[\begin{array}{l}
\boldsymbol{\theta} \\
\boldsymbol{\phi}
\end{array}\right]
$$

The output equations for a set of sensors, represented by the matrix $\mathbf{L}_{\mathbf{y}}$, can be defined by

$$
\mathbf{y}=\mathbf{L}_{y}^{T} \mathbf{q}=\mathbf{L}_{y}^{T} \boldsymbol{\Psi} \boldsymbol{\eta}=\boldsymbol{\Psi}_{y} \boldsymbol{\eta} .
$$

The state-space equations can be written by (8) and (9)

$$
\begin{gathered}
\dot{\mathbf{x}}=\left[\begin{array}{cc}
\mathbf{0} & \mathbf{I} \\
-\boldsymbol{\Omega}^{2} & -2 \boldsymbol{\xi} \Omega
\end{array}\right] \mathbf{x}+\left[\begin{array}{c}
\mathbf{0} \\
\boldsymbol{\mu}^{-1} \boldsymbol{\Psi}_{u}^{T}
\end{array}\right] \mathbf{f} \\
\mathbf{y}=\left[\begin{array}{ll}
\boldsymbol{\Psi}_{\mathbf{y}} & \mathbf{0}
\end{array}\right] \mathbf{x}
\end{gathered}
$$

where $\mathbf{x}=\left[\begin{array}{ll}\boldsymbol{\eta} & \dot{\boldsymbol{\eta}}\end{array}\right]^{\mathbf{T}}$ are the state variables.

This model-reduction technique is applied to extract several reduced models for discrete configurations.

\section{Affine Models-Interpolation and Simulation}

A set of reduced single-input single-output (SISO) models, which can be built from the procedure described in the previous section, is used to build an LPV model in the configuration space:

$$
\begin{aligned}
& \dot{\mathbf{x}}=\mathbf{A}(\mathbf{l}) \mathbf{x}+\mathbf{B}(\mathbf{l}) \mathbf{u} \\
& \mathbf{y}=\mathbf{C}(\mathbf{l}) \mathbf{x}+\mathbf{D}(\mathbf{l}) \mathbf{u}
\end{aligned}
$$

where $\mathbf{x}$ is the state of the system, $\mathbf{u}$ and $\mathbf{y}$ are the input and the output, respectively, and $\mathbf{l}$ is a vector of varying parameters. In order to keep the conventional state-space notation, the input $\mathbf{u}=\mathbf{f}$ is adopted.

The technique used to create the LPV model relies on a linear interpolation of discrete poles, zeros, and gains [16], [20]. This approach is suitable for models with few poles and zeros, justifying the model reduction step. This fact is not a limiting factor, since few poles and zeros are usually sufficient to represent the motion of a mechatronic system within the frequency range of interest. Equation (11) illustrates the technique applied for the vector of poles:

$$
\left[\begin{array}{c}
p_{1}(\mathbf{l}) \\
p_{2}(\mathbf{l}) \\
\vdots \\
p_{n}(\mathbf{l})
\end{array}\right]=\left[\begin{array}{c}
p_{0,1} \\
p_{0,2} \\
\vdots \\
p_{0, n}
\end{array}\right]+\left[\begin{array}{c}
p_{1,1} \\
p_{1,2} \\
\vdots \\
p_{1, n}
\end{array}\right] f(\mathbf{l})
$$

where $p_{1}$ till $p_{n}$ are the poles of the system, $p_{0,1}$ till $p_{0, n}$ and $p_{1,1}$ till $p_{1, n}$ are constants, and $f(\mathbf{l})$ is an analytical function of the scheduling parameter $\mathbf{l}$. Similar affine functions have been made to describe the varying zeros and gains. Based on these affine functions, proper first- and second-order state-space subsystems can be derived.

From a pair of complex poles $\left(p_{i}(\mathbf{l}), p_{i+1}(\mathbf{l})\right)$ and a pair of zeros $\left(z_{i}(\mathbf{l}), z_{i+1}(\mathbf{l})\right)$, a second-order state-space subsystem can be derived by:

$$
\begin{aligned}
& \mathbf{A}_{s}^{2}(\mathbf{l})=\mathbf{R e}\left[\begin{array}{cc}
p_{i}(\mathbf{l})+p_{i+1}(\mathbf{l}) & -p_{i}(\mathbf{l}) p_{i+1}(\mathbf{l}) \\
1 & 0
\end{array}\right] \\
& \mathbf{B}_{s}^{2}(\mathbf{l})=\left[\begin{array}{l}
1 \\
0
\end{array}\right] \\
& \mathbf{C}_{s}^{2}(\mathbf{l})=\operatorname{Re}\left[\begin{array}{c}
-z_{i}(\mathbf{l})-z_{i+1}(\mathbf{l})+p_{i}(\mathbf{l})+p_{i+1}(\mathbf{l}) \\
z_{i}(\mathbf{l}) z_{i+1}(\mathbf{l})-p_{i}(\mathbf{l}) p_{i+1}(\mathbf{l})
\end{array}\right]^{T} \\
& \mathbf{D}_{s}^{2}(\mathbf{l})=[1] .
\end{aligned}
$$


From one pole $(p(\mathbf{l}))$ and one zero $z(\mathbf{l})$, a first-order state-space subsystem can be derived by:

$$
\begin{gathered}
\mathbf{A}_{s}^{1}(\mathbf{l})=[p(\mathbf{l})] \quad \mathbf{B}_{s}^{1}(\mathbf{l})=[1] \\
\mathbf{C}_{s}^{1}(\mathbf{l})=[p(\mathbf{l})-z(\mathbf{l})]^{T} \quad \mathbf{D}_{s}^{1}(\mathbf{l})=[1] .
\end{gathered}
$$

These subsystems are then concatenated, yielding a varying state-space model. The concatenation can be performed between two subsystems $\left(\mathbf{A}_{s 1}, \mathbf{B}_{s 1}, \mathbf{C}_{s 1}, \mathbf{D}_{s 1}\right)$ and $\left(\mathbf{A}_{s 2}, \mathbf{B}_{s 2}, \mathbf{C}_{s 2}\right.$, $\left.\mathbf{D}_{s 2}\right)$ :

$$
\begin{array}{rlrl}
\mathbf{A}_{c} & =\left[\begin{array}{cc}
\mathbf{A}_{s 2} & \mathbf{0} \\
\mathbf{B}_{s 1} \mathbf{C}_{s 2} & \mathbf{A}_{s 1}
\end{array}\right] & \mathbf{B}_{c}=\left[\begin{array}{c}
\mathbf{B}_{s 2} \\
\mathbf{B}_{s 1} \mathbf{D}_{s 2}
\end{array}\right] \\
\mathbf{C}_{c}=\left[\begin{array}{ll}
\mathbf{D}_{s 1} \mathbf{C}_{s 2} & \mathbf{C}_{s 1}
\end{array}\right] & \mathbf{D}_{c}=\left[\mathbf{D}_{s 1} \mathbf{D}_{s 2}\right] .
\end{array}
$$

All subsystems can then be concatenated, yielding an LPV statespace system that is quadratically dependent on the affine function $f(\mathbf{l})$ [16]. The gain is eventually added to the system.

This LPV model can be implemented in Simulink using S-functions. For each integration step, the S-functions compile the state-space model from the LPV model depending on the parameter vector.

\section{Test CASE: Pick-AND-Place Assembly Robot}

In this section, the pick-and-place assembly robot and its model are described. The model is then experimentally validated.

\section{A. Description of the Pick-and-Place Assembly Robot Setup}

The chosen test case is an industrial three-axis pick-andplace machine (see Fig. 1). The $Y$-motion is gantry driven by two linear motors and the $X$-motion over the carriage is also driven by a linear motor. The vertical $Z$-motion is actuated by a rotary brushless dc motor that drives a vertical beam by a ball screw/nut combination. The position of the linear motors and the beam length are measured with optical encoders and the acceleration at the gripper in the $X$-direction is measured with an accelerometer.

The objective of a pick-and-place machine is to move the gripper as accurate and fast as possible in a point-to-point motion. However, fast movements of the linear motor will excite the eigenfrequencies of the flexible beam, which may vary during the movement, since the beam length is continuously changed.

\section{B. Modeling}

A model has been built to simulate the pick-and-place assembly robot motion in $X$ - and $Z$-direction. The $Y$-motion is not considered in this paper. According to the proposed modeling methodology, the system is divided in two subsystems:

1) a subsystem whose dynamics does not depend on the configuration; and

2) a subsystem with configuration-dependent dynamics.

The subsystem containing the frame, the two linear motors that drive the $Y$-motion, the carriage, their bushings, and joints does not depend on the configuration and is modeled

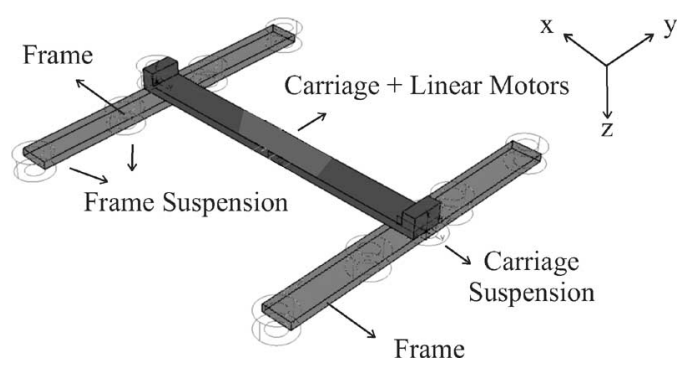

Fig. 3. Multibody model of the subsystem that does not depend on the configuration.

in LMS Virtual.Lab Motion, a commercial multibody environment. Fig. 3 shows the multibody model of this subsystem.

The total mass of the frame and of both linear motors (which drives the $Y$-motion) is $169 \mathrm{~kg}$. The carriage weighs $13.9 \mathrm{~kg}$. These values can be found in the machine manual. The frame suspension is attached to the ground by eight connecting points. The frame suspension stiffness and the damping constant are $2.6 \mathrm{e} 7 \mathrm{~N} / \mathrm{m}$ and $2500 \mathrm{Ns} / \mathrm{m}$, respectively. A spring and a damper represent the connection between the carriage and the frame and their values are $9.2 \mathrm{e} 6 \mathrm{~N} / \mathrm{m}$ and $1000 \mathrm{Ns} / \mathrm{m}$, respectively. The stiffness and damping values are adjusted to match the experimental data.

The dynamics of the beam and the linear motor that drives the $X$-motion depend on configuration and are modeled using the aforementioned three-step methodology. First, a parametric high-order finite-element model of this subsystem is created. Fig. 2(A) shows a scheme of the parametric dependence of the model for several discrete positions. There is one rigid mode, the lateral motion on $X$-direction. In this figure, the connection between the linear motor and the beam is modeled as a linear stiffness $K=2.5 \mathrm{e} 11 \mathrm{~N} / \mathrm{m}, M=25 \mathrm{~kg}$ represents the motor mass, $x$ the rigid-mode degree of freedom and $m_{i}=\left(0.53-l_{i}\right) \pi r^{2} \rho$ the equivalent mass of the part of the beam that is connected to the linear motor. The gripper mass is $m_{g}=1.2 \mathrm{~kg}$. The nominal beam radius is $r=0.012 \mathrm{~m}$. The material properties of the beam are: density $\rho=7800 \mathrm{~kg} / \mathrm{m}^{3}$, Poisson's ratio $\nu=0.3$, damping ratio 0.01 , and elasticity modulus $E=2.12 \mathrm{e} 11 \mathrm{~N} / \mathrm{m}^{2}$. The beam length, $l_{i}$, can vary from 0.33 to $0.53 \mathrm{~m}$. A parametric finiteelement model is created using the Structural Dynamics Toolbox (SDT) in Matlab. For each configuration, a finite-element model was derived from the parametric one modifying the beam length. The flexible beam is modeled with 100 beam elements, which guarantees good accuracy for the finite-element model and for the reduced model.

The suggested model-reduction technique is applied for 20 finite-element models representing 20 configurations (between 0.33 and $0.53 \mathrm{~m}$ ). These reduced models are then interpolated in order to generate the LPV model. There is no rule to decide the amount of configurations that should be evaluated. However, it is important to highlight that the proposed interpolation technique is based on the linear relation between the scheduling parameter and the system poles and zeros; therefore, this fact should be verified in advance. In this case study, the poles and the zeros of these 20 configurations seem to vary in a linear 
(a)
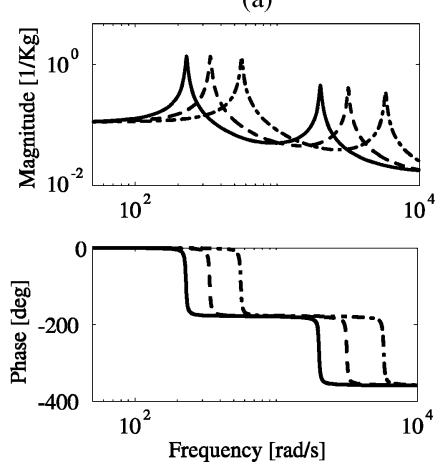

(b)
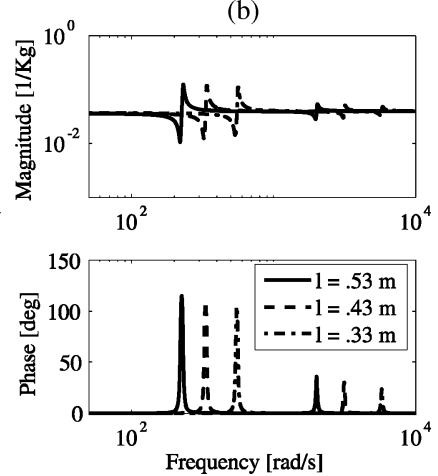

Fig. 4. (a) $\mathbf{a}_{g} / F_{m}$. (b) $\mathbf{a}_{m} / F_{m}$ for $l=0.53,0.43,0.33 \mathrm{~m}$.

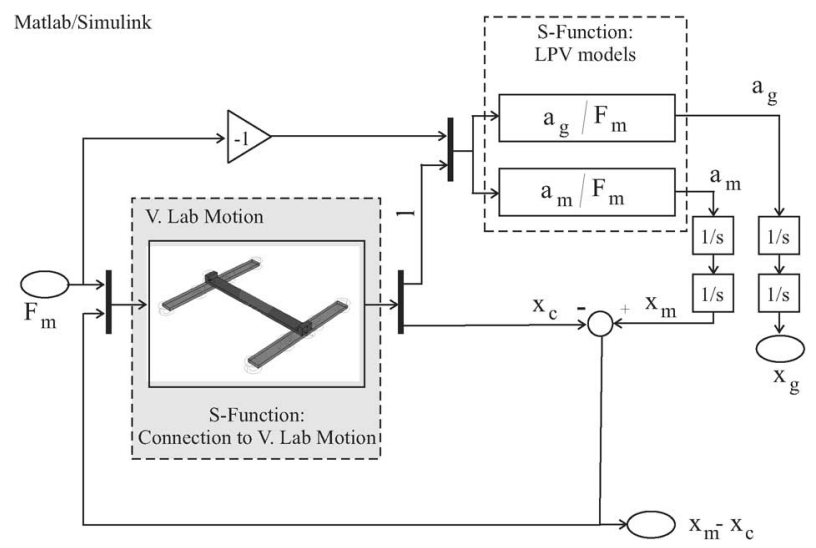

Fig. 5. Cosimulation scheme between LMS Virtual.Lab Motion (gray background) and Matlab/Simulink (white background).

way according to the configuration. Higher-order interpolations should be considered if the poles and the zeros seem not to vary in a linear way (see [20]).

Each model has 1 degree of freedom for the actuator (linear motor) and two modal coordinates, representing the first and the second natural frequencies. This single-input multiple-output (SIMO) model has the force applied by the motor as input, $\mathbf{F}_{m}$, and the gripper and motor accelerations as outputs, $\mathbf{a}_{g}$ and $\mathbf{a}_{m}$, respectively. Fig. 4 illustrates frequency response functions (FRFs) of the system at three different beam lengths $(1=[0.53$, $0.43,0.33 \mathrm{~m}])$.

These SIMO models are divided into two SISO models since the interpolation technique can only be applied for a set of SISO models. The proposed interpolation technique has been applied yielding two SISO LPV models. The chosen analytical function $f(\mathbf{l})$ is $\mathbf{l}$, the measurement of the beam length since a linear relation between the subsystem eigenfrequencies and the beam length can be verified in Fig. 4. S-functions have been implemented in Matlab/Simulink in order to simulate these LPV SISO models (see Fig. 5).

There are two inputs for these S-functions: the force of the linear motor, $\mathbf{F}_{m}$, and the beam length, l. For each integration step, the S-functions compile the ABCD-model from the LPV model depending on the beam length.
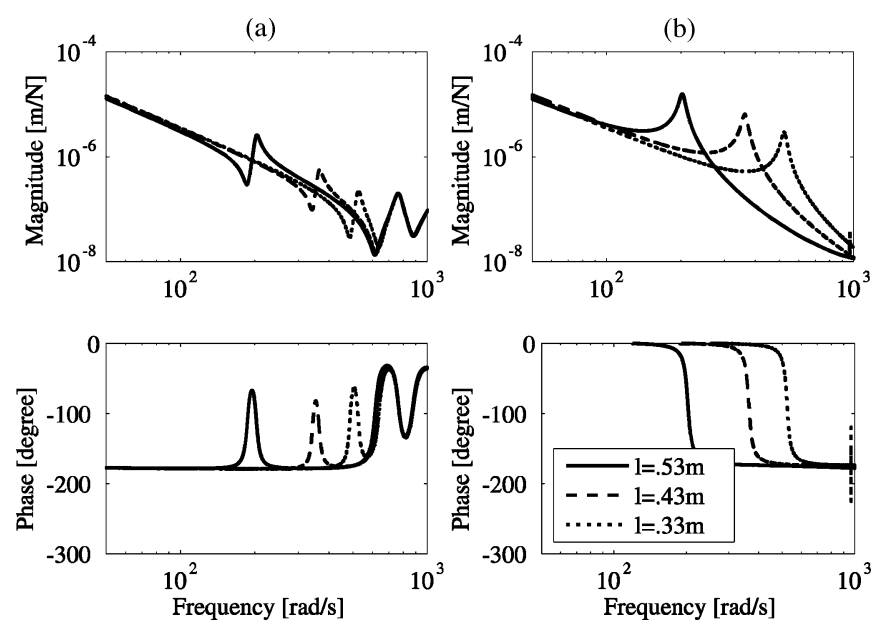

Fig. 6. (a) $\left(\mathbf{x}_{m}-\mathbf{x}_{c}\right) / F_{m}$. (b) $\mathbf{x}_{g} / F_{m}$ for $l=0.53,0.43,0.33 \mathrm{~m}$.

The LMS Virtual.Lab Motion interface with Matlab/Simulink allows time-domain simulations of arbitrary mechanisms coupled with transfer functions or state-space models described in Matlab/Simulink. Therefore, both subsystems are integrated through cosimulation between LMS Virtual.Lab Motion and Matlab/Simulink (see Fig. 5). The force generated by the motor, $\mathbf{F}_{m}$, is applied to the motor (S-function input in Matlab/ Simulink for the LPV model) and to the carriage (in Motion) with opposite directions (action and reaction forces). The encoder of the motor measures the difference between the positions of the motor, $\mathbf{x}_{m}$ (S-function output in Matlab/Simulink for the LPV model), and the carriage, $\mathbf{x}_{c}$ (in Motion). The motor and the gripper positions, $\mathbf{x}_{m}$ and $\mathbf{x}_{g}$, are calculated by integrating twice the motor and the gripper acceleration, $\mathbf{a}_{m}$ and $\mathbf{a}_{g}$, respectively. The FRFs obtained using this approach for three beam lengths are depicted in Fig. 6.

The controllers of the $Y$-motion and the $X$-motion are independent; therefore, they can be designed separately. The description of the $Y$-motion modeling is omitted because the main concern, in this paper, is to control the $X$-motion. The $Y$-motion modeling can be performed using the same methodology since it also depends on the configuration. The system division would be performed in a similar way: the subsystem (1) would consist of the carriage, the linear motors, and the flexible beam; and the subsystem (2) would be the frames.

\section{Experimental Validation}

In order to validate the model, comparisons between simulated and experimental results were performed. The setup is identified for four different lengths of the beam, based on FRF measurements using multisine excitation [20], [21]. The linear motor responsible for the $X$-direction motion is excited with a multisine voltage signal with random phases. Two measurements are acquired: the encoder position, $\mathbf{x}_{m}-\mathbf{x}_{c}$, and the gripper acceleration, $\mathbf{a}_{g}$. An average over five experiments with different realizations of the random phases is performed in order to obtain the FRFs. 

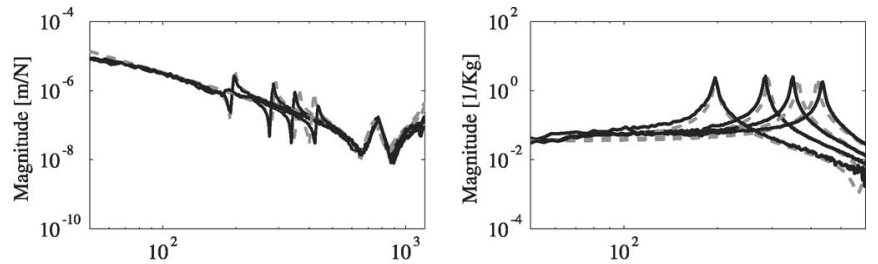

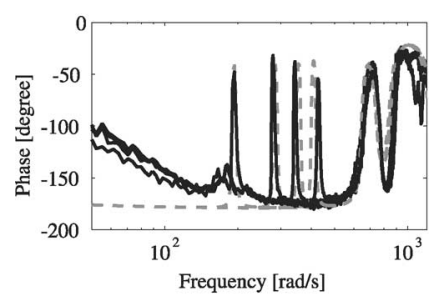

(a)

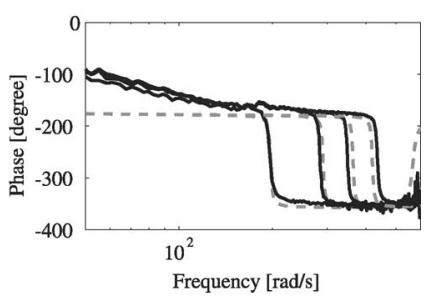

(b)
Fig. 7. Comparison between the simulated (- - dashed line) and the experimental FRFs (- full line). (a) $\left(\mathbf{x}_{m}-\mathbf{x}_{c}\right) / F_{m}$. (b) $\mathbf{a}_{g} / F_{m}$.
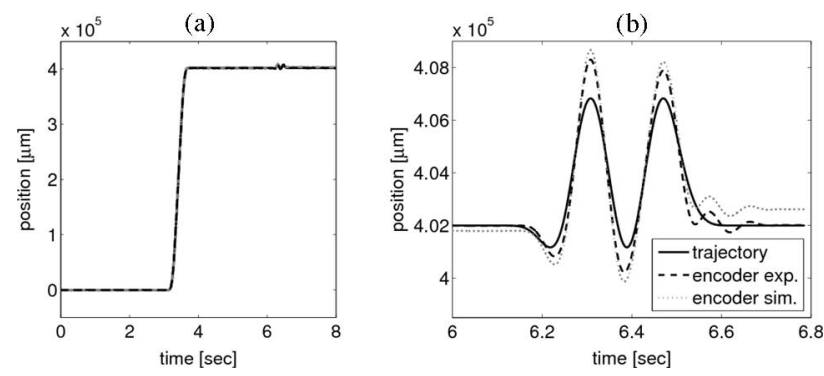

Fig. 8. Encoder (motor position): comparison between the simulated and the experimental motor position for the same current input. (a) From 0 to $8 \mathrm{~s}$. (b) From 6 to $6.8 \mathrm{~s}$.

Fig. 7 shows the comparisons for four beam lengths $(1=$ $0.53,0.41,0.36$, and $0.33 \mathrm{~m}$ with the first resonance frequency at 32, 45, 56, and $66 \mathrm{~Hz}$, respectively). The comparisons are similar for other beam lengths. Differences at low frequencies are mainly due to the lack of friction in the model and differences in high frequency are mainly due to a mismatch in the sensor position; since in the model, it is placed on the end of the flexible beam, and in the setup, it is place on the gripper. For controlling the motion of mechatronic systems, such as machine tools and mechanisms, a control bandwidth around $30 \mathrm{~Hz}$ is typically expected (as discussed in Section IV-A). Consequently, a model able to capture accurately the machine dynamics up to $100 \mathrm{~Hz}$ is usually sufficient. The proposed model can be used for control design and evaluation.

Since the approach generates a new state-space model for each time-integration step, it is possible to perform a trajectory with continuously varying beam length. A value proportional to the measured current input was used as input signal to the model since the current and the motor force are typically proportional for a linear motor. The encoder measurements obtained by the experiment and by the simulation are compared in Fig. 8. In this experiment, the beam length does not change significantly (about $20 \%$ of the total range) since, in the actual machine, the velocity of the $X$-motion is much higher than the velocity of the $Z$-motion. However, even this limited range analysis can assure a confident correlation between the model and the real system since the dynamics vary quite smoothly.

\section{MEChATRONIC DESIGN APPROACH}

A mechatronic design approach has been employed considering as variables: the radius of the flexible beam (discrete, values: $0.010,0.012,0.016$, and $0.020 \mathrm{~m}$ ) and the gains of the PID controller (continuous). An optimal PID controller is designed for each possible radius of the flexible beam. Therefore, structural and control parameters are being considered and tradeoff designs can be evaluated for several active systems in a truly mechatronic design approach.

By choosing the control strategy a priori, the size of the optimization problem is reduced. LTI and LPV control strategies are exploited hereafter for designing a PID controller for the $X$ direction motion. It is necessary to highlight that the $Z$-direction motion is added to the model, but no control design is carried out for it, since its dynamics do not depend on the configuration. The $Z$-direction movement is considered ideal, i.e., the applied motion is perfectly tracked.

The following sections describe the chosen control design approach based on time-domain metrics, the LTI PID, controllers and LPV PID controllers. Design tradeoffs considering structural and control parameters are highlighted for both control strategies.

\section{A. Control Design Approach}

During the control specification, time- and/or frequencydomain characteristics are used to evaluate closed-loop performance. The FRFs of the open-loop and closed-loop transfer functions, such as sensitivity and complementary sensitivity functions, are typically used to characterize closed-loop performance [22]. The common frequency-domain metrics are gain and phase margins (GM and PM, respectively), the maximum value of sensitivity and complementary sensitivity functions $\left(M_{S}\right.$ and $M_{T}$, respectively). It is easier to evaluate the feedback performance in the frequency domain. However, for time-variant systems, such as mechatronic systems with configuration-dependent dynamics, frequency-domain metrics are not ready available since there is no plant that represents the system for all simulation time and/or configurations.

The idea in this paper is to design a controller for a timevariant system using the total variation (TV), a time-domain metric, to evaluate the performance of the system. The TV of a function $g(t)$ can be defined as the largest sum of variations for any subdivision of $t$ [22]. Considering a step response (see Fig. 9), the TV and the excess variation ( $E V)$ [22] can be defined as:

$$
\mathrm{TV}=\sum_{i} v_{i}, \quad \mathrm{EV}=\frac{T V}{v_{0}}
$$

In the cases considered hereafter, $v_{0}=1$, then $\mathrm{TV}=\mathrm{EV}$.

The TV was chosen rather than the setting time and the overshoot since it offers a tradeoff between these two conflicting time-domain metrics. Using frequency-domain performance criteria, an upper bound in $M_{T}$ has been a frequent 


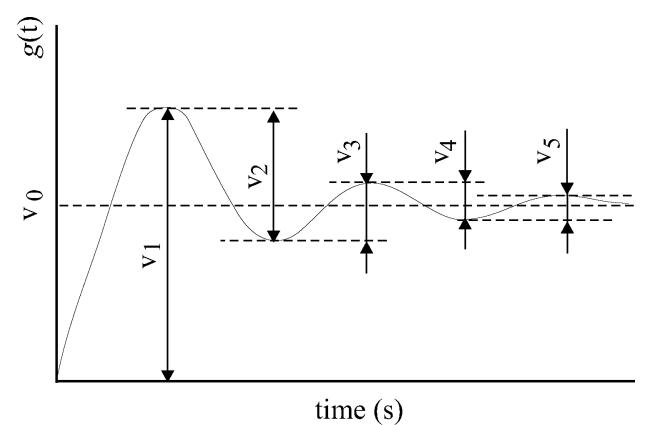

Fig. 9. Total variation (TV) and excess variation (EV) of $g(t)$.

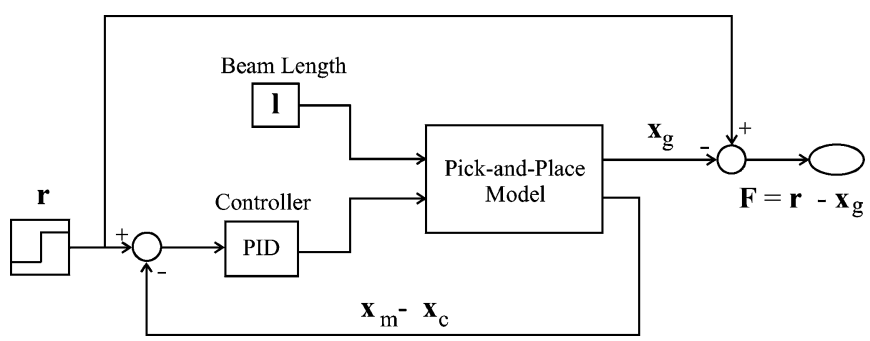

Fig. 10. Simulink model used for the optimization loop.

design specification. Indeed, $M_{T}<2$ guarantees $G M \geq 1.5$ and $P M \geq 29^{\circ}$ [22]. The TV of the step response in the time domain can be quite well correlated to $M_{T}$ and will be used as a criterion to evaluate the performance of the system [22].

$$
M_{T} \leq T V \leq(2 n+1) M_{T}
$$

where $n$ is the order of the complementary sensitivity function. Most of the system dynamics can be represented by low-order models, suggesting that TV provides a good approximation to $M_{T}$ [22]. Consequently, TV should be smaller than 2 and as close as possible to 1 , in order to guarantee good performance.

The optimization problem is described by a multiobjective problem where the difference between the reference input, $\mathbf{r}$, and the position of the gripper, $\mathbf{x}_{g}$, should be minimized for all $m$ time steps during the simulation time (see in Fig. 10).

$$
\min _{\mathbf{S} \in \Re^{n}} f(\mathbf{S})=\frac{1}{2} \sum_{i=1}^{m}\left(r_{i}(\mathbf{S})-x_{g i}((\mathbf{S}))\right)^{2}=\frac{1}{2} \sum_{i=1}^{m} F_{i}(\mathbf{S})^{2}
$$

where the $\mathrm{n} \times 1$ vector $\mathbf{S}$ is the variables vector that describes the PID gains. The beam radius are considered as a discrete parameter in Sections IV-B and IV-C.

This optimization problem was solved using LevenbergMarquardt optimization method. This method combines the steepest descent method with Gauss-Newton method [23], aiming to find $\mathbf{S}=\left\{s_{1}, s_{2}, \ldots, s_{n}\right\}^{T}$ that minimizes a least-squares problem, $f(\mathbf{S})$ :

$$
\min _{\mathbf{S} \in \Re^{n}} f(\mathbf{S})=\frac{1}{2} \sum_{i=1}^{m} F_{i}(\mathbf{S})^{2}
$$

TABLE I

OPTIMAL LTI PID AND TV FOR DIFFERENT BEAM LENGTHS AND NOMINAL RADIUS $(0.012 \mathrm{~m})$

\begin{tabular}{|c||c|c|c||c|c||}
\hline $\begin{array}{c}\text { Beam } \\
\text { length }[m]\end{array}$ & $\begin{array}{c}K_{P} \\
{\left[\frac{N}{\mu m}\right]}\end{array}$ & $\begin{array}{c}K_{I} \\
{\left[\frac{N}{\mu m \cdot s}\right]}\end{array}$ & $\begin{array}{c}K_{D} \\
{\left[\frac{N \cdot s}{\mu m}\right]}\end{array}$ & $\begin{array}{c}T V 1 \\
{[\mu m]}\end{array}$ & $\begin{array}{c}T V 2 \\
{[\mu m]}\end{array}$ \\
\hline 0.53 & 0.461 & 0.068 & 0.007 & 1.17 & 3.22 \\
\hline 0.43 & 1.078 & 0.028 & 0.009 & 1.17 & 3.72 \\
\hline 0.33 & 1.813 & 0.000 & 0.011 & 1.20 & 3.60 \\
\hline
\end{tabular}
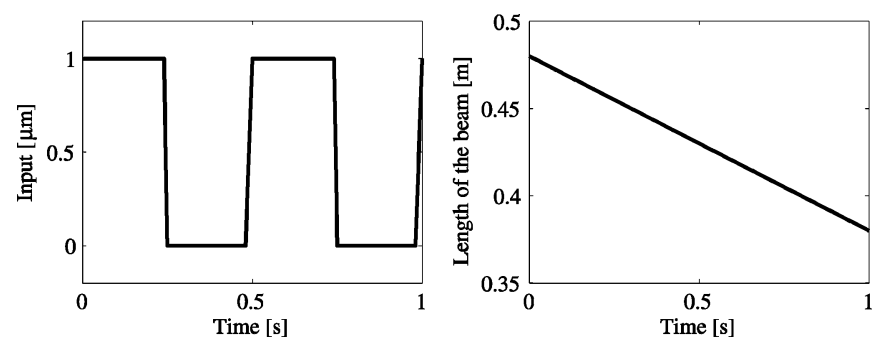

Fig. 11. Pulse input while the length of the beam is continuously changed.

where $F_{i}(\mathbf{S})$ are the objective functions. The solution can be found iteratively:

$$
\mathbf{S}_{i+1}=\mathbf{S}_{i}-\left(\mathbf{J}\left(\mathbf{S}_{i}\right)^{T} \mathbf{J}\left(\mathbf{S}_{i}\right)+\lambda_{i} \mathbf{I}\right)^{-1} \mathbf{J}\left(\mathbf{S}_{i}\right)^{T} f\left(\mathbf{S}_{i}\right)
$$

where $\mathbf{J}$ is the Jacobian matrix of the function $f(\mathbf{S}), \mathbf{I}$ is the identity matrix, and $\lambda_{i}$ is a constant that ensures the positive definiteness of $\left(\mathbf{J}\left(\mathbf{S}_{i}\right)^{T} \mathbf{J}\left(\mathbf{S}_{i}\right)+\lambda_{i} \mathbf{I}\right)$. This method has been largely used for tracking problems and parameter estimation [23].

\section{B. LTI PID Controllers}

First, the optimization problem was solved for a fixed radius $(0.012 \mathrm{~m})$, considering a step input as a reference signal. Table I shows the gains of the optimal PID $\left(K_{P}, K_{I}, K_{D}\right)$ resulted from the optimization for different beam lengths. The TV for the motor position measured by the encoder (TV1) and for the gripper position (TV2) are also shown.

As it can be observed in Table I that the optimal PID controllers for different lengths are not the same. In order to choose an LTI PID controller for the system, considering the beam length variation, a pulse train was chosen as input while the beam length was continuously changed, according to Fig. 11. The optimization problem is carried out in the same way, using (17).

The flexible beam is a commercially available product and its radius can be $0.010,0.012,0.016$, and $0.020 \mathrm{~m}$. The optimal LTI PID gains and the TVs (TV1 and TV2) for the nominal radius and for the commercial available ones is shown in Table II. Clearly, there is a tradeoff between the positioning of the motor and the vibration of the gripper. Thinner beams lead to better motor position performance and worse gripper position performance than thicker beams. Considering that the TV of the gripper should be as close as possible to 2, the best design option would be the thicker beam $(0.020 \mathrm{~m})$. However, considering the structural and control design space, no design guarantees $\mathrm{GM} \geq 1.5$ and $\mathrm{PM} \geq 29^{\circ}$, i.e., TV2 $>2$ for all possible structural modification. 
TABLE II

OPTIMAL LTI PID GAINS AND TV FOR DIFFERENT RADIUS

\begin{tabular}{|c||c|c|c||c|c||}
\hline $\begin{array}{c}\text { Radius } \\
{[m]}\end{array}$ & $\begin{array}{c}K_{P} \\
{\left[\frac{N}{\mu m}\right]}\end{array}$ & $\begin{array}{c}K_{I} \\
{\left[\frac{N}{\mu m \cdot s}\right]}\end{array}$ & $\begin{array}{c}K_{D} \\
{\left[\frac{N \cdot s}{\mu m}\right]}\end{array}$ & $\begin{array}{c}T V 1 \\
{[\mu m]}\end{array}$ & $\begin{array}{c}T V 2 \\
{[\mu m]}\end{array}$ \\
\hline 0.010 & 0.761 & 0.010 & 0.012 & 1.10 & 4.62 \\
\hline 0.012 & 1.091 & 0.085 & 0.010 & 1.15 & 3.80 \\
\hline 0.016 & 1.783 & 0.075 & 0.011 & 1.23 & 3.11 \\
\hline 0.020 & 2.180 & 0.201 & 0.011 & 1.26 & 2.45 \\
\hline
\end{tabular}

TABLE III

LTI, INTERPOLATED LPV AND OPTIMIZED LPV PID GAINS AND TV FOR THE NOMINAL RADIUS $(0.012 \mathrm{~m})$

\begin{tabular}{|c||c|c|c|c||c|c||}
\hline Methodology & $\begin{array}{c}\text { index } \\
{[0,1]}\end{array}$ & $\begin{array}{c}K_{P} \\
{\left[\frac{N}{\mu m}\right]}\end{array}$ & $\begin{array}{c}K_{I} \\
{\left[\frac{N}{\mu m \cdot s}\right]}\end{array}$ & $\begin{array}{c}K_{D} \\
{\left[\frac{N \cdot s}{\mu m}\right]}\end{array}$ & $\begin{array}{c}T V 1 \\
{[\mu m]}\end{array}$ & $\begin{array}{c}T V 2 \\
{[\mu m]}\end{array}$ \\
\hline \hline time-invariant & - & 1.091 & 0.085 & 0.010 & 1.15 & 3.80 \\
\hline interpolation & 0 & 4.044 & -0.011 & 0.017 & 1.22 & 3.85 \\
& 1 & -6.760 & 0.340 & -0.020 & & \\
\hline \multirow{2}{*}{ optimization } & 0 & 4.044 & -0.011 & 0.015 & 1.16 & 2.72 \\
\hline
\end{tabular}

\section{LPV PID Controllers}

Another alternative to cope with tighter performance requirements for this time-variant problem is by using an LPV controller. In this way, the control can be modified according to the configuration. First, a gain-scheduling controller is created linearly interpolating the optimized gains for discrete configurations (20). For the nominal beam radius $(0.012 \mathrm{~m})$, the gains are linearly interpolated from the three configurations shown in Table I. The results obtained using this approach are shown in Table III. The indexes 0 and 1 represent the constant coefficients of the linear interpolation function, as described for the proportional gain:

$$
K_{P}=K_{P 0}+l K_{P 1} .
$$

As it can be noticed, the LPV PID controller, using linearly interpolated gains, does not present any performance improvement as compared to the LTI PID controller using the optimized gains over the chosen trajectory, shown in Table II.

These interpolated LPV PID gains may be a good "hot start" for an optimization problem considering the LPV PID gains ( $K_{P 0}, K_{P 1}, K_{I 0}, K_{I 0}, K_{D 0}$, and $\left.K_{D 1}\right)$ as variables. The gains calculated from this optimization are refereed to as the optimized LPV gains and the gains calculated from the interpolation are refereed to as the interpolated LPV gains. Table III shows that the controller using the optimized LPV gains achieved better performance than the interpolated LPV gains and LTI PID gains. Moreover, it can be observed that by choosing the optimized LPV PID, a thinner beam $(0.012 \mathrm{~m})$ yields similar performance than the best design option $(0.020 \mathrm{~m})$ when the LTI PID is considered (see Table II).

Finally, Table IV shows the TVs, TV1 and TV2, for each commercially available flexible beam when the LPV PID gains are optimized. For the sake of brevity, the gains are omitted. The same tradeoff design between the positioning of the motor and the vibration of the gripper can be verified: thinner flexible beams lead to better motor position performance and worse gripper position performance than thicker flexible beams; however, using the LPV PID controller, tighter performance requirements
TABLE IV

TV FOR DIFFERENT RADIUS CONSIDERING THE OPTIMIZED LPV PID CONTROLLER

\begin{tabular}{|c||c|c||}
\hline $\begin{array}{c}\text { Radius } \\
{[\mathrm{m}]}\end{array}$ & $\begin{array}{c}T V 1 \\
{[\mu \mathrm{m}]}\end{array}$ & $\begin{array}{c}T V 2 \\
{[\mu \mathrm{m}]}\end{array}$ \\
\hline 0.010 & 1.11 & 3.65 \\
\hline 0.012 & 1.16 & 2.72 \\
\hline 0.016 & 1.23 & 2.32 \\
\hline 0.020 & 1.27 & 2.15 \\
\hline
\end{tabular}

can be fulfilled. This relations can only be verified through simulation during the design phase.

\section{CONCLUSION}

A methodology to model and simulate mechatronic systems with configuration-dependent dynamics, using affine reduced models and cosimulation, was presented. This methodology was applied to a pick-and-place assembly robot. The system was divided into two subsystems: a subsystem whose dynamics do not depend on the configuration and a subsystem with configurationdependent dynamics. The former was modeled using LMS Virtual.Lab Motion and the latter using an LPV model derived from the linear interpolation of reduced finite-element models. Both the subsystems are coupled using cosimulation. Since the cosimulation environment is Matlab/Simulink, the methodology allows also the inclusion of the control actions in the simulation. The model was experimentally validated demonstrating that this methodology is able to provide a good approximation of the real behavior of a mechatronic system with configuration-dependent dynamics.

Using the simulation capabilities, integrated design was performed considering control and structural parameters. These results exemplify the benefits of the mechatronic design approach since the active system design tradeoffs were identified. For instance, in closed-loop simulation, thinner flexible beams lead to better motor position performance and worse gripper position performance than thicker flexible beams. The advantage of using the mechatronic design approach is to enable the evaluation, not only of the qualitative behavior, such as the previous analysis, but also quantitative metrics, such as the TV values.

Finally, using time-domain metrics, LTI PID, and LPV PID controllers were designed for each possible structural modification. It has been observed that better performance is reached using the LPV PID controller derived from an optimization problem, which minimizes the TV of the gripper position and considers the PID gains as variables. Therefore, the same performance can be reached by smaller diameters using LPV controllers, as by thicker diameters using LTI controllers.

\section{ACKNOWLEDGMENT}

The authors would like to thank the reviewers for their constructive comments and suggestions.

\section{REFERENCES}

[1] W. Symens, H. Van Brussel, and J. Swevers, "Gain-scheduling control of machine tools with varying structural flexibilities," Ann. CIRP, vol. 52 no. 1, pp. 321-324, 2004. 
[2] P. R. Pagilla, B. Yu, and K. L. Pau, "Adaptive control of time-varying mechanical systems: Analysis and experiments," IEEE/ASME Trans. Mechatronics, vol. 5, no. 4, pp. 410-418, Dec. 2000.

[3] H. Van Brussel, P. Sas, I. Nemeth, P. De Fonseca, and P. Van den Braembussche, "Towards a mechatronic compiler," IEEE/ASME Trans. Mechatronics, vol. 6, no. 1, pp. 90-105, Mar. 2001.

[4] W. J. Zhang, Q. Li, and L. S. Guo, "Integrated design of mechanical structure and control algorithm for a programmable four-bar linkage," IEEE/ASME Trans. Mechatronics, vol. 4, no. 4, pp. 354-362, Dec. 1999.

[5] Z. Affi, B. EL-Kribi, and L. Romdhane, "Advanced mechatronic design using a multi-objective genetic algorithm optimization of a motor-driven four-bar system," Mechatronics, vol. 17, pp. 489-500, 2007.

[6] T. Ravichandran, D. Wang, and G. Heppler, "Simultaneous plantcontroller design optimization of a two link planar manipulator," Mechatronics, vol. 16, pp. 233-242, 2006.

[7] P. De Fonseca, "Simulation and optimisation of the dynamic behaviour of mechatronic systems," Ph.D. thesis, Katholieke Univ. Leuven, Leuven, Belgium, 2000.

[8] F. Wujun and Z. Changming, "Active damping guide roller design based on integrated structure/controller optimization with a structured controllers," in Proc. ASME 2005 Int. Des. Eng. Tech. Conf. Comput., Inf. Eng. Conf., Long Beach, CA, Sep., pp. 1-5.

[9] S. Behabahani and C. W. de Silva, "System-based and concurrent design of a smart mechatronic system using the concept of mechatronic design quotient (MDQ)," IEEE/ASME Trans. Mechatronics, vol. 13, no. 1, pp. 14-21, Feb. 2008.

[10] L. P. R. de Oliveira, M. M. da Silva, P. Sas, H. Van Brussel, and W. Desmet, "Concurrent mechatronic design approach for active control of cavity noise," J. Sound Vib., vol. 314, no. 3-5, pp. 507-525, 2008.

[11] L. Zollo, S. Roccella, E. Guglielmelli, M. Chiara Carrozza, and P. Dario, "Biomechatronic design and control of an anthropomorphic artificial hand for prosthetic and robotic applications," IEEE/ASME Trans. Mechatronics, vol. 12, no. 4, pp. 418-429, Aug. 2007.

[12] X. Wang and J. K. Mills, "Dynamic modeling of a flexible-link planar parallel platform using substructuring approach," Mech. Mach. Theory, vol. 41, pp. 671-687, 2006

[13] M. Zaeh and D. Siedl, "A new method for simulation of machining performance by integrating finite element and multi-body simulation for machine tools," Ann. CIRP, vol. 56, no. 1, pp. 383-386, 2007.

[14] A. Konno, M. Uchiyama, and M. Murakami, "Configuration-dependent vibration controllability of flexible-link manipulators," Int. J. Robot. Res., vol. 56, no. 4, pp. 567-576, 1997.

[15] X. Wang and J. K. Mills, "Active control of configuration-dependent linkage vibration with application to a planar parallel platform," in Proc. 2005 IEEE Int. Conf. Robot. Autom., Barcelona, Spain, Apr., pp. 43274332.

[16] B. Paijmans, W. Symens, H. Van Brussel, and J. Swevers, "A gainscheduling-control technique for mechatronic systems with positiondependent dynamics," in Proc. Amer. Control Conf., Minneapolis, MN, Jun. 14-16, 2006, pp. 2933-2938, Paper ThB04.4.

[17] O. A. Bauchau and C. L. Bottasso, "Contact conditions for cylindrical, prismatic, and screw joints in flexible multibody systems," Multibody Syst. Dyn., vol. 5, no. 3, pp. 251-278, 2001.

[18] R. R. Craig, "A review of time domain and frequency domain component mode synthesis methods," in Proc. Joint Mech. Conf., Albuquerque, NM, Jun. 24-26, 1985, pp. 1-30.

[19] A. Preumont, Vibration Control of Active Structures: An Introduction. 2nd ed. Dordrecht, The Netherlands: Kluwer, 2002.

[20] B. Paijmans, W. Symens, H. Van Brussel, and J. Swevers, "Identification of interpolating affine LPV models for mechatronic systems with one varying parameter," Eur. J. Control, vol. 14, no. 1, pp. 16-29, 2008.
[21] J. Schoukens and R. Pintelon, System Identification: A Frequency Domain Approach. Piscataway, NJ: IEEE Press, 2001.

[22] S. Skogestad and I. Postlethwaite, Multivariable Feedback Control : Analysis and Design. Chichester, U.K.: Wiley, 1997.

[23] J. Nocedal and S. Wright, Numerical Optimization. New York: SpringVerlag, 2000.

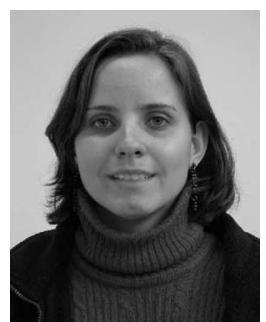

Maíra M. da Silva received the Bachelors' and Masters' degrees in mechanical engineering from the University of São Paulo, São Paulo, Brazil, in 2001 and 2004, respectively. She is currently working toward the Ph.D. degree in the Department of Mechanical Engineering, Katholieke Universiteit Leuven, Leuven, Belgium.

Her current research interests include flexible multibody systems, vehicle dynamics, modeling of mechatronic system, motion and vibration control, and optimization.

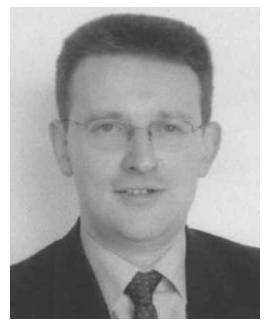

Wim Desmet received the M.Sc. and Ph.D. degrees in mechanical engineering from Katholieke Universiteit (K.U.) Leuven, Leuven, Belgium, in 1992 and 1998, respectively.

$\mathrm{He}$ is currently a Professor with the Noise and Vibration Research Group, K.U. Leuven. His current research interests include numerical and experimental vibro-acoustics, uncertainty modeling of dynamic systems, aeroacoustics, active noise and vibration control, noise control engineering, multibody dynamics, vehicle technology, and virtual prototyping.

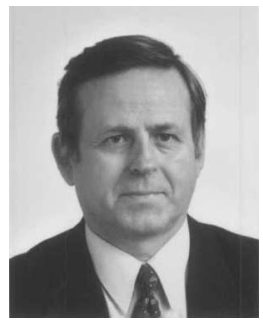

Hendrik Van Brussel (S'68-M'68-SM'85-F'95) received the B.Sc. ME (Technisch Ingenieur) degree from Hoger Technisch Instituut, Oostende, Belgium, in 1965, and the M.Sc. EE (Burgerlijk Ingenieur) and $\mathrm{Ph} . \mathrm{D}$. degrees from Katholieke Universiteit (K.U.) Leuven, Leuven, Belgium, in 1968 and 1971, respectively.

$\mathrm{He}$ is currently a Professor in Mechatronics and Automation at K.U. Leuven. His current research interests include mechatronics (motion and vibration control, precision engineering, the mechatronic compiler), robotics (behavior-based control, medical robotics, mobile manipulation, industrial robotics, service robots), and holonic manufacturing systems (multiagent systems, biologically inspired coordination, and control systems). 